Acta Crystallographica Section D

Biological Crystallography

ISSN 0907-4449

\section{Thomas Gutberlet, ${ }^{\mathrm{a} *}$ Udo Heinemann $^{\mathrm{b}, \mathrm{c}}$ and Michael Steiner $^{\mathrm{a}}$}

${ }^{a}$ Hahn-Meitner-Institut Berlin, Department SF1, Glienicker Strasse 100, 14109 Berlin, Germany, b Max-Delbrück-Center for Molecular Medicine, Department of Crystallography, Robert-RössleStrasse 10, 13122 Berlin, Germany, and 'Institute of Chemistry - Crystallography, Free University of Berlin, Takustrasse 6, 14195 Berlin, Germany

Correspondence e-mail: gutberlet@hmi.de

\title{
Protein crystallography with neutrons - status and perspectives
}

The current state of the art and future perspectives for protein crystallography with neutrons have been reviewed and discussed at an international workshop recently organized by the Hahn-MeitnerInstitut (HMI) and Max-Delbrück-Center (MDC) in Berlin, and supported by the Neutron Round Table activity. The main topics discussed and the conclusions drawn are presented in this paper. While the available flux from even the most powerful neutron sources is much lower than that available at a modern synchrotron-radiation source, there is no doubt that neutrons can additionally provide vital information in structural biology that is difficult to obtain using other methods. One of the overriding themes at the workshop was the fact that the current instrumentation and facilities do not fully exploit the neutron beams that are available. Current trends indicate that many of the problems that have limited the efficiency of biological neutron scattering have been recognized and with new technological advances important efforts are under way to tackle these problems. It is clear that the use of neutrons in biology will become increasingly significant at existing and new neutron sources.
Received 14 September 2000 Accepted 7 December 2000

\section{Introduction}

The progress in the Human Genome Project and other structural genomics projects is dramatically changing the research strategies used to study and understand complex biochemical pathways and disease processes. In order to develop a comprehensive mechanistic understanding of physiological processes at the molecular and atomic levels, new strategies and possibilities in structural biology have to be applied in order to organize the information explosion provided by the various genome projects. Protein structure determination by X-rays, electron microscopy, NMR and neutrons will play a central role in this context (Sali, 1998). Several initiatives, such as the 'Protein Structure Initiative' of the National Institute of General Medical Sciences (Zapp Machalek, 2000), the 'New York Structural Genomics Research Consortium' (Burley, 2000) and the German structural genomics project 'Proteinstrukturfabrik' (Bork, 2000; Heinemann et al., 2000), aim at highthroughput programs for protein structure determination on a fast, efficient and routine basis.

In recent years, such routine analysis of protein structures has been performed moreor-less exclusively at synchrotron-radiation sources using high-performance detector systems and cryoprotected small samples. Highly resolved crystal structures are obtained with a resolution of $\sim 1.5 \AA$ and in many cases better $(\sim 3 \%$ of all current structures in the Protein Data Bank). The location of $\mathrm{H}$ atoms in proteins, nucleic acids and sometimes even water molecules, essential in understanding and determining reaction pathways and structure-function relationships in biological systems, is achieved only at the highest resolutions of about $1 \AA$ and below and where the relevant atoms are ordered sufficiently well.

The $\mathrm{H}$ atoms, an essential part of the complete structure, can be localized more easily by neutron diffraction (without the demanding requirements for very high resolution data) owing to the negative neutronscattering length $b_{\mathrm{H}}$ of hydrogen and its relative strength of scattering with respect to $\mathrm{C}, \mathrm{N}$, $\mathrm{O}$ etc. and the unique possibility of contrast variation by exchange with deuterium with a large positive $b_{\mathrm{D}}$ (Schoenborn \& Knott, 1996). Consequently, neutrons can be used to determine the position of $\mathrm{H}$ atoms, water molecules or disordered molecules such as surfactants within a protein crystal which scatter too weakly to be detected by X-rays alone. Thus, neutron diffraction should be a versatile tool in protein structure determination. In fact, however, neutron diffraction has been used only rarely in this field. Only 36 entries
C 2001 International Union of Crystallography Printed in Denmark - all rights reserved 
containing the keyword 'neutron' are listed in the Protein Data Bank at Rutgers University out of a total of 11563 entries at the beginning of the year 2000. Why does neutron diffraction not have a broader impact on protein structure determination, if neutrons have the potential to localize proton positions so well?

Historically, the main drawback of using neutron diffraction in protein structure determination has been the requirement for relatively large protein crystals of several cubic millimetres and the long dataacquisition times of up to weeks per data set owing to the available neutron flux even at high-power neutron sources as the ILL. Recent progress in improving Laue diffraction, new neutron optics, new detector technologies and longer neutron wavelengths now show dramatic changes in these problems, with possible huge gains in efficiency. Samples of volume $1 \mathrm{~mm}^{3}$ are now treatable for small proteins $(\sim 15 \mathrm{kDa})$.

Current efforts worldwide to upgrade existing neutron sources and to build new ones such as the Spallation Neutron Source (SNS) in the USA (Moncton, 2000), the proposed Joint Neutron Project in Japan (Furusaka \& Oyama, 1999) and the proposed European Spallation Source (ESS) in Europe (Kjems et al., 1997) will offer higher flux neutron beams with optimized signal-to-noise ratio for the time-offlight Laue method and, in parallel, efforts to develop new and to upgrade existing neutron instruments for protein crystallography will change the unsatisfactory situation and open up more reliable and new possibilities for neutron diffraction in this field of structural research.

An international workshop recently organized by the Hahn-Meitner-Institut (HMI) and Max-Delbrück-Center (MDC) in Berlin on 15-16 February 2000, and supported by the European Commission Traning and Mobility of Researchers (EC-TMR) Neutron Round Table activity, reviewed and discussed the current state of the art and future perspectives in protein crystallography with neutrons. The main topics raised and discussed and the conclusions drawn are briefly presented in the following report.

\section{Localization of $\mathbf{H}$ atoms and water molecules by neutron diffraction}

The main questions to be solved using neutron diffraction are firstly the position of the $\mathrm{H}$ atoms (as deuterium) and of water or surfactant molecules within a protein crystal which are disordered or scatter too weakly to be detected with X-rays at sufficient resolution. Secondly, knowing the position of the $\mathrm{H}$ atoms and thereby defining hydrogen-bonding patterns and the protonation state of catalytic residues plays a fundamental role in the mechanistic understanding of enzyme reactivity. Examples that may serve to illustrate the second point include the ribonucleases, where the protonation state of histidine residues is of crucial importance, and glycosyl hydrolases such as $\beta$-glucanases, whose catalytic action depends on the protonation state of activesite carboxylates (Pace et al., 1991; Heinemann et al., 1996). However, H-atom positions are difficult to define with X-rays, even at resolutions between 1.2 and $1 \AA$, often considered 'atomic' resolution. Recent examples proving this point include the X-ray structures of a synthetic RNA duplex determined at $1.16 \AA$ (Mueller, Schübel et al., 1999; Mueller, Muller et al. 1999) and of the cold shock protein $B c$-sp of Bacillus caldolyticus determined at $1.17 \AA$ resolution (Mueller et al., 2000). Successful detection of such $\mathrm{H}$-atom positions by X-rays depends crucially on the individual $B$ factors.

An increase in the resolution of the X-ray structure determination to better than $1.2 \AA$ is required to localize $\mathrm{H}$-atom positions. At one extreme, for example, the Bacillus lentus subtilisin, at a resolution of $0.78 \AA$, shows many polar $\mathrm{H}$ atoms, including some establishing the bonding pattern in an internal water channel of the protein (Kuhn et al., 1998). However, considering the effort necessary to achieve such exceptionally high resolution in X-ray structure determination of proteins, the question has been raised whether efforts towards solving this problem by neutron diffraction (i.e. growing large crystals) might be less time-consuming and more profitable.

For enzymes, the classical neutron study in this field is that of Kossiakoff \& Spencer (1981) on trypsin. Extending to larger molecular weight proteins (beyond $15 \mathrm{kDa}$ ) required the improvements in equipment and source referred to above. In neutron Laue diffraction, neutron study of $25 \mathrm{kDa}$ proteins was pioneered in the investigation of the sugar-binding plant lectin concanavalin A by Habash et al. (1997). In a second study (Habash et al., 2000) using neutron Laue diffraction, the bound water $\mathrm{D}_{2} \mathrm{O}$ molecules at $2.4 \AA$ (unit-cell volume $480000 \AA^{3}$ ) were assigned; $62 \mathrm{D}_{2} \mathrm{O}$ molecules and 20 with one $\mathrm{D}$ atom were visible even at room temperature. In the ultrahighresolution X-ray case (Deacon et al., 1997), only $12 \mathrm{H}_{2} \mathrm{O}$ molecules, although 35 with one $\mathrm{H}$ atom visible, were resolved. In addi- tion, the three bound waters' D atoms at the saccharide-binding site of concanavalin A were revealed, which is a significant improvement in the structural definition of bound water molecules at a ligand site and a new level of detail for molecular-recognition and molecular-modelling studies (Habash $e t$ al., 2000).

The potential of neutron diffraction for the localization of $\mathrm{H}$ atoms at the catalytic centre of an enzyme is demonstrated by recent work on the complex of the aspartic proteinase endothiapepsin (Cooper \& Myles, 2000). The structure of the inhibitor at the catalytic centre was resolved by neutron Laue diffraction at $1.95 \AA$ resolution, resolving the location of the crucial protons and identifying buried negatively charged carboxylate groups, both not observed by X-ray diffraction at around $2 \AA$ resolution. By deuteration of the active site, non-negatively charged Asp residues could be identified.

The location of water molecules in the light-driven proton pump bacteriorhodopsin (Hauss et al., 1997), the displacement of water molecules during the pumping cycle (Weik, Zaccai et al., 1998) and conformational changes in the tertiary structure have been investigated in recent years by detailed neutron diffraction work (Dencher et al., 1989). In addition, the orientation and location of retinal and the assignment of $\alpha$-helices in the structure were also determined using neutrons. Only recently, the structure of the proton pathway in the cytoplasmic domain of bacteriorhodopsin was also obtained by X-ray diffraction with a resolution of $2.25 \AA$ (Sass et al., 2000). However, the dynamics of the retinal in bacteriorhodopsin were established by inelastic neutron scattering (Reat et al., 1998); such experiments would have been extremely difficult with X-rays.

An outstanding example of the localization and refinement of $\mathrm{H}$-atom positions using neutrons has been provided by the structural refinement of cyclodextrin inclusion complexes (Saenger \& Steiner, 1998). Owing to the availability of large crystals, neutron diffraction at very high resolution, better than $1 \AA$, enabled the characterization in detail of the properties of dynamically disordered networks of conventional and non-conventional hydrogen bonds $(\mathrm{C}-\mathrm{H} \cdots \mathrm{O}, \mathrm{C}-\mathrm{H} \cdots \pi)$ in such systems (Steiner \& Saenger, 1993).

Unique information on the location of $\mathrm{H}$ atoms and water has also been obtained by neutron fibre diffraction of biological polymers such as cellulose (Nishiyama et al., 1999), hyaluronic acid (Deriu et al., 1997), 
filamentous viruses (Langan, 1997; Mitsch, 1998) and DNA (Pope, Forsyth et al., 1998; Pope, Shotton et al., 1998). For example, individual water positions along the DNA strand have been refined even at the low resolution of $3 \AA$ (Shotton et al., 1997).

Another aspect tackled by neutron diffraction is the localization of surfactants added to proteins. The detergent structure in integral membrane proteins has not been possible to determine in X-ray diffraction measurements owing to the disorder of the surfactants in the unit cell. Matching the scattering-length density of the protein by appropriate addition of $\mathrm{D}_{2} \mathrm{O}$ visualized the surfactant. Thus, even at low-resolution neutron diffraction of $12-16 \AA$ it was possible to resolve the detergent structure in the OmpF porin of Escherichia coli or the porin of Rhodobacter capsulatus (PebayPeyroula et al., 1995; Penel et al., 1998). Contrast variation also allows dense water shells around proteins to be determined by low-resolution neutron diffraction or the interaction of proteins with glycolipid head groups, important in signal transduction (Weik, Patzelt et al., 1998). Given that $40 \%$ of a genome is membrane-bound proteins, which are extremely difficult to crystallize, the strategic importance of such neutron scattering studies in revealing the interactions of protein and detergent cannot be emphasized enough.

The aforementioned examples in the use of neutrons in protein structure determination highlight a few important selected topics where the method has been applied recently. Successful applications have in general been based on the availability of large protein crystals, e.g. the crystal in the $2.43 \AA$ resolution neutron Laue diffraction study of the sugar-binding state in concanavalin A was about $1.7 \mathrm{~mm}^{3}$ (Habash et al., 2000). This is about 85 times larger in volume than the average $0.02 \mathrm{~mm}^{3}$ crystals used for the $0.94 \AA$ X-ray measurements (Deacon et al., 1997). Nevertheless, the results of the localization of protons (preferably as deuterons) and the determination of protein-ligand binding structures clearly demonstrate the feasibility of the use of neutrons in protein crystallography. The current efforts in the development of new instruments and the upgrading of existing instruments can therefore be hoped to result in significant improvements in the use of neutrons in this field.

A further development of huge potential to widen the technique is the preparation of perdeuterated proteins from bacteria grown on perdeuterated media. The incoherent hydrogen background scattering is avoided and the signal-to-noise ratio at the edge of neutron diffraction patterns is improved. Resolution limits can thus be extended compared with incompletely deuterated proteins.

\section{Neutron instrumentation for protein crystallography}

At present, the number of instruments in the world that can be used for protein neutron crystallography is rather limited. Most research is concentrated on instruments at the ILL (Grenoble). There, for classical single-crystal diffractometry with monochromated neutrons the neutron four-circle diffractometers D19, DB21 and D16 have been successfully used for collecting protein crystallographic data, and a new Laue diffractometer with cold neutrons (cold LADI) is now dedicated to protein crystallography.

D19 is a thermal monochromatic neutron diffractometer optimized for samples with medium-sized unit cells. It can operate at a number of wavelengths in the range 1.0 $2.4 \AA$ (Forsyth et al., 1998). The instrument is equipped with a two-dimensional multi-wire detector that has an aperture of $4^{\circ}$ in the horizontal plane by $64^{\circ}$ in the vertical plane. The diffractometer is routinely used for structure determination of small molecules of biological interest but has also been used to study vitamin $\mathrm{B}_{12}$ (Bouquiere et al., 1994), lysozyme (Lehmann et al., 1985, 1989) and haemoglobin (Waller, 1989). It is also currently the only instrument in the world that can carry out high-angle fibre diffraction work and has produced excellent results on hydration patterns around polymeric DNA (Pope, Forsyth et al., 1998; Pope, Shotton et al., 1998) and hydrogen-bonding patterns in different forms of cellulose (Nishiyama et al., 1999). Other systems that have been studied in this way include hyaluronic acid (Deriu et al., 1997) and filamentous viruses (Langan, 1997; Mitsch, 1998). The limited size of the current area detector means that this instrument is underexploited by a factor of at least 20 . Proposals to purchase a large array of detectors that will solve this problem are currently in progress (ILL's Millennium Refurbishment programme). The work on DNA (Pope, Forsyth et al., 1998; Pope, Shotton et al., 1998) has illustrated the advantages that are possible for biological neutron diffraction by sample deuteration. Likewise, Shu et al. (1996) have demonstrated the benefits of perdeuterated myoglobin.
DB21 is a cold neutron diffractometer developed by ILL and EMBL-Grenoble for low-resolution $(>10 \AA)$ neutron protein crystallography of complex biological macromolecules (i.e. multimeric proteins or assemblies of proteins with nucleic acids such as viruses or ribosomes). Here, the aim is to locate disordered components in the crystal that cannot be observed by X-ray crystallography alone. This is achieved by contrast-variation techniques in which data are collected at a series of different $\mathrm{H}_{2} \mathrm{O}$ / $\mathrm{D}_{2} \mathrm{O}$ crystal solvent concentrations (or contrasts) that match and cancel the scattered signal from each component in turn. In some cases, the visibility of specific components can be further enhanced by selective deuteration prior to crystallization (e.g. the detergent used in the case of a membrane protein). The technical challenges of collecting very low resolution data are overcome by using long-wavelength neutrons $(7.5 \AA)$ and the resolution of the instrument is adapted to large unit-cell edges of up to $1000 \AA$, opening a wider potential applicability.

The four-circle diffractometer D16 is used for the study of biological membranes and structures of about $50 \AA$ periodicity that diffract to a real-space resolution of several angstroms. The instrument is equipped with a position-sensitive multidetector with $64 \times$ 16 wires. The detector can be turned by $90^{\circ}$ to place the best angular resolution in either the horizontal or vertical direction. It can be scanned around the sample to observe diffraction out to an angle of about $120^{\circ}$. With its high-intensity focused beam it is ideal for the study of lamellar or in-plane scattering from membrane systems. A large body of data from purple membrane (Weik, Zaccai et al., 1998; Dencher et al., 1989) as well as lamellar scattering from phospholipid model membranes has been obtained from this instrument.

An instrument of comparable design, the cold neutron diffractometer V1 at HMI (Berlin) is optimized for the study of lamellar arranged systems such as stacked multilayers of bacteriorhodopsin or phospholipids. Equipped with a high-resolution area detector which provides a sensitive area of $20 \times 20 \mathrm{~cm}$ with a spatial resolution of $1.5 \times 1.5 \mathrm{~mm}$, monochromatic wavelengths between 3 and $6 \AA$ are used, increasing the scattering efficiency at longer wavelengths. On the instrument, the position of essential water molecules in bacteriorhodopsin in the ground and $\mathrm{M}$ states, respectively, was studied (Weik et al., 1998) and the number of water molecules in the ground state was determined (Hauss et al., 1997). Recently, 
data on the photosynthetic reaction centre of Rhodobacter sphaeroidis were obtained with a theoretical resolution of $15 \AA$ (Hauss, 2000). Unfortunately, owing to the limited neutron flux of the HMI reactor, the measurement time to collect sufficient data for structure analysis would be several months, but the diffractometer is nevertheless very useful for analyzing protein crystal quality and for determining lowresolution structure data.

With the development of neutronsensitive image plates, a new generation of advanced two-dimensional protein neutron diffractometers have been built that are dedicated to neutron protein crystallography at high resolution (1.5 $\AA$ ). The first neutron Laue diffractometer LADI was realised at ILL (Grenoble) using a white neutron beam. The combination of a broadbandpass quasi-Laue geometry with a novel $2 \pi$ neutron-sensitive image-plate detector to record long-wavelength neutrons up to $4 \AA$ (Cipriani et al., 1996) provides 10-100-fold gains in data-collection rates compared with the conventional neutron diffractometers (Habash et al., 1997; Helliwell, 1997; Myles et al., 1998; Niimura et al., 1997). The instrument operates either in Laue mode, using a full white neutron beam, or more commonly in a quasi-Laue mode, where $\mathrm{Ti} / \mathrm{Ni}$ multilayer band-pass filters are used to tune the wavelength and select the bandpass $(d \lambda / \lambda=8-30 \%)$ of the incident beam to match the demands of the sample. The instrument is used for single-crystal studies of small proteins up to $25-30 \mathrm{kDa}$ at medium $(2.4 \AA)$ to high resolution $(2 \AA)$ in order to locate individual $\mathrm{H}$ atoms of special interest, water structures or other small molecules that can be marked by deuterium to be particularly visible. The size of the unit cell that can be studied depends on the size of the crystal; beyond $\sim 300000 \AA^{3}$ crystals of several cubic millimetres are required. First experiments provided detailed information on hydration states in tetragonal and triclinic crystal forms of lysozyme determined at $2.0 \AA$ (Niimura et al., 1997) and $1.7 \AA$ (Bon et al., 1999), respectively, on carbonyl residues of concanavalin A (Habash et al., 1997) and on the neutron structure of enzyme vitamin $\mathrm{B}_{12}$ at $1.45 \AA$ (Langan et al., 1999). Recent studies involved the aspartic protease endothiapepsin (Cooper \& Myles, 2000) referred to above. For concanavalin A (Habash et al., 1997, 2000), hydrogen/deuterium locations determined at medium resolution $(\sim 2.4 \AA)$ on LADI have been shown to provide information on many bound water molecules that could not be determined from

Table 1

Neutron instruments for protein crystallography.

\begin{tabular}{|c|c|c|c|}
\hline Instrument & Facility & Description & Contact \\
\hline D19 & ILL & $\begin{array}{l}\text { Thermal neutron four-cycle diffractometer, } \\
\text { wavelength range } 1.0-2.4 \AA\end{array}$ & http://193.49.43.3/YellowBook/D19/ \\
\hline DB21 & ILL & $\begin{array}{l}\text { Cold neutron low-resolution diffractometer, } \\
\text { wavelength } 4.6 \text { and } 7.56 \AA\end{array}$ & http://193.49.43.3/YellowBook/DB21/ \\
\hline D16 & ILL & $\begin{array}{l}\text { Cold neutron two- or four-circle diffractometer, } \\
\text { wavelength } 4.5 \text { and } 5.6 \AA\end{array}$ & http://193.49.43.3/YellowBook/D16/ \\
\hline $\mathrm{V} 1$ & HMI & $\begin{array}{l}\text { Cold neutron two-circle diffractometer, } \\
\text { wavelength range } 3.0-6.0 \AA\end{array}$ & $\begin{array}{l}\text { http://www.hmi.de/bensc/instrumentation/ } \\
\text { instrumente/v1/v1.html }\end{array}$ \\
\hline LADI & ILL & Cold neutron Laue diffractometer & http://193.49.43.3/YellowBook/LADI \\
\hline BIX-3 & JAERI & $\begin{array}{l}\text { Diffractometer for monochromated neutrons, } \\
\text { wavelength } 2.2 \AA\end{array}$ & $\begin{array}{l}\text { Advanced Science Research Center, JAERI, } \\
\text { Tokai-mura, Ibaraki, 319-1195, Japan }\end{array}$ \\
\hline SXD & ISIS & $\begin{array}{l}\text { Time-of-flight Laue diffractometer, } \\
\text { wavelength range } 2 \text { to } 10 \AA\end{array}$ & http://www.isis.rl.ac.uk/crystallography/sxd.htm \\
\hline
\end{tabular}

atomic resolution $(<1.1 \AA)$ synchrotron X-ray data alone (Deacon et al., 1997).

At the Japanese neutron facility JAERI (Tokai-mura), a combination of a singlecrystal diffractometer BIX-3 with a monochromatic thermal neutron beam and a neutron-sensitive image plate was realised for the investigation of protein crystals (Niimura, 1996). Monochromatizing the neutron beam by a bent perfect Si crystal, the protein crystals are analyzed by a crystal-step scan method $\left(\sim 0.2^{\circ}\right.$ rotation between each 5 min image) to produce highresolution diffraction patterns. Although the data-collection efficiency is inferior to that of quasi-Laue techniques, the background is less and therefore, at the expense of long measuring times, higher resolution data can be recorded (Niimura et al., 1997). Thanks to a high reduction of the $\gamma$-sensitivity of the neutron image plate used at the instrument, the signal-to-noise ratio has been further improved enormously. High-resolution data well below $2 \AA$ - with virtually no overlap of single Bragg reflections - can be collected at this instrument.

In contrast to the above diffractometers at reactor-based neutron sources with a continuous neutron flux, spallation-based neutron sources have a time-dependent neutron flux and single-crystal diffractometry is performed in the time-of-flight mode, which allows background noise to be largely discriminated out by the counter electronics. This method is applied on the single-crystal diffractometer SXD at ISIS, which uses the time-of-flight Laue technique to access large volumes of reciprocal space in a single measurement (Keen \& Wilson, 1996). Exploitation of the flexible datacollection method offered by this technique allows data sets to be collected in relatively short periods of time, typically in the range 1-2 s per diffraction pattern for small organic molecules. Using a wavelength range of $0.2-10 \AA$, three fibre-optically encoded ZnS scintillators, each with $192 \times$ $192 \mathrm{~mm}$ active area and $3 \times 3 \mathrm{~mm}$ resolution, are used for data acquisition. An increase in the number of detectors (to 11) is funded and under construction. Also, a reconfiguration of the whole instrument is proposed to facilitate neutron protein crystallography at ISIS for the first time with relocation of the diffractometer to a cold neutron beam, beam focusing and an increase in the number of detectors. A second new dedicated time-of-flight singlecrystal diffractometer for protein crystallography is also planned on the proposed second target station of ISIS.

Currently, the Biosciences Division of the Los Alamos National Laboratory is building a time-of-flight single-crystal diffractometer at LANSCE. This instrument is dedicated to protein, membrane and fibre diffraction (Schoenborn, 1996). This diffractometer will operate in the wavelength range $1-5 \AA$ achieving a neutron flux at the sample of $\sim 7 \times 10^{6}$ neutrons $\mathrm{s}^{-1} \mathrm{~cm}^{-2}$ using a partially coupled moderator (Schoenborn et al., 1999). The position-sensitive $\mathrm{He}^{3}$ cylindrical detector will cover $2000 \mathrm{~cm}^{2}$ with a resolution of $1.3 \mathrm{~mm}$ FWHM and a counting rate $>10^{6}$ neutrons s ${ }^{-1}$ (Schoenborn, 1992). To prevent spot overlap and improve the signalto-noise ratio, a chopper system will eliminate the initial radiation pulse and provide a short- and long-wavelength cutoff. The effective neutron flux of this instrument will gain at least a factor of about four in comparison with a similar reactor-based instrument (Schoenborn, 1996), thus reducing the data-acquisition time.

\section{Conclusions and outlook}

The seven neutron instruments currently running worldwide (Table 1) which are used in protein crystallography demonstrate the feasibility of exploring protein structures with the use of neutrons. It is clear that 
progress in this field has been hampered significantly owing to the severe restrictions in sample size; results of general scientific interest have mainly been achieved only at the most powerful neutron source, the ILL, as illustrated in this report. During the workshop the following were described: (i) recent progress in the development of neutron image-plate detector systems, (ii) efforts to improve the existing instruments and (iii) the possibilities of spallation-based neutron sources with future increased neutron flux and enhanced signal-to-noise ratio from the time-of-flight approach, demonstrating important efforts in the further and future application of neutrons in protein crystallographic research. A challenge to prove and expand this perspective would be furthered by the construction of a dedicated single-crystal diffractometer for protein crystallography at least at one of the proposed and/or new neutron-spallation sources worldwide, i.e. ESS or SNS, respectively.

In addition, it must also be mentioned that progress in sample preparation with enlarged increased crystal sizes by the development of standard protein crystallization techniques and sophisticated use of deuterated samples to enhance the signal-tonoise ratio in neutron-scattering experiments will further benefit the approach. Deuterium labelling has not been a routine technique for many structural biologists up to now, but is becoming widely used in NMR applications. At the workshop the general opinion was expressed that neutrons, as a complementary tool for determining water and H-atom positions (Helliwell, 1997) and in understanding intramolecular and intermolecular interactions in proteins, can gain more interest in the protein community. This interest will also grow with the announced increase in the number of protein structure determinations arising from structural genomics projects (Sali, 1998). It will be further amplified if the sample-size requirements for neutron experiments fall to lower values, also circumventing the problems of extreme cooling of large crystallites, which has so far been rarely, if ever, applied. Progress in this field is foreseeable by the efforts described in instrument improvements and by the availability of more powerful neutron sources.

Nevertheless, in the near future the overall number of entries in the Protein Data Bank related to neutrons will grow only slowly. Synchrotron-based protein crystallography is by far, and will remain, the more efficient strategy for simple structure determination. However, as discussed at the workshop, it has to be stated that for the solution of dedicated problems related to the visualization of hydrogen, such as the definition of protonation states of catalytic residues, affinities in ligand-protein binding or details of bound water or hydrogen bonding, neutrons are a powerful and uniquely qualified tool and usually yield critical information. In addition, sophisticated information about the dynamics of proteins and water can be applied using inelastic neutron scattering, a topic beyond protein structure determination and not discussed at the workshop, although referred to briefly (for a current review on this subject, see Byron \& Gilbert, 2000).

In efficient research strategies, scientists will take into account these aspects and will exploit the complementary and unique possibilities of the neutron as a probe as far as is possible according to its availability.

The authors would like to thank for valuable comments and discussions Trevor Forsyth, John R. Helliwell, Dave Keen, Dean Myles, Werner Press and Peter Timmins. Support from the ECU-TMR Neutron Round Table activity is also acknowledged. At the workshop, presentations were given by Georg Büldt (FZ Jülich), Jon Cooper (University of Southampton), Trevor Forsyth (ILL), Thomas Hauss (HMI), Udo Heinemann (MDC), John R. Helliwell (University of Manchester), Dave Keen (ISIS), Dean Myles (EMBL), Nobuo Niimura (JAERI), Benno P. Schoenborn (LANL), Thomas Steiner (FU Berlin), Peter Timmins (ILL) and Joe Zaccai (CEA/ CNRS).

\section{References}

Bon, C., Lehmann, M. S. \& Wilkinson, C. (1999). Acta Cryst. D55, 978-987.

Bork, P. (2000). Biophys. J. 78, 19A

Bouquiere, J. P., Finney, J. L. \& Savage, H. F. J. (1994). Acta Cryst. B50, 566-578.

Burley, S. K. (2000). Biophys. J. 78, 19A.

Byron, O. \& Gilbert, R. J. C. (2000). Curr. Opin. Biotechnol. 11, 72-80.

Cipriani, F., Castagna, J. C., Wilkinson, C., Oleinek, P. \& Lehmann, M. S. (1996). J. Neutron Res. 4, 79-85.

Cooper, J. B. \& Myles, D. A. A. (2000). Acta Cryst. D56, 246-248.

Deacon, A., Gleichmann, T., Kalb (Gilboa), A. J., Price, H., Raftery, J., Bradbrook, G., Yariv, J. \& Helliwell, J. R. (1997). J. Chem. Soc. Faraday Trans. 93, 4305-4312.

Dencher, N. A., Dresselhaus, D., Zaccai, G. \& Büldt, G. (1989). Proc. Natl Acad. Sci. USA, 86, 7876-7879.

Deriu, A., Cavatorta, F., De Micheli, T., Rupprecht, A. \& Langan, P. (1997). Physica B, 234-236, 215-216.
Forsyth, V. T., Shotton, M. W., Ye, H., Boote, C., Langan, P., Pope, L. H. \& Denny, R. C. (1998). Fibre Diffraction Rev. 7, 17-24.

Furusaka, M. \& Oyama, Y. (1999). Contributions to the 6th ESS General Meeting, edited by F. Carsughi, A. Fournier \& H. Ullmaier. Jülich: ESS Council.

Habash, J., Raftery, J., Nuttall, R., Price, H. J., Wilkinson, C., Kalb (Gilboa), A. J. \& Helliwell, J. R. (2000). Acta Cryst. D56, 541-550.

Habash, J., Raftery, J., Weisgerber, S., Cassetta, A., Lehmann, M. S., Hoghoj, P., Wilkinson, C., Campbell, J. W. \& Helliwell, J. R. (1997). J. Chem. Soc. Faraday Trans. 93, 4313-4317.

Hauss, T. (2000). Personal communication.

Hauss, T., Papadopoulos, G., Verclas, A. A. W., Büldt, G. \& Dencher, N. (1997). Physica B, 234236, 217-219.

Heinemann, U., Aÿ, J., Gaiser, O., Müller, J. J. \& Ponnuswamy, M. N. (1996). Biol. Chem. 377, 447-454.

Heinemann, U., Frevert, J., Hofmann, K.-P., Illing, G., Oschkinat, H., Saenger, W. \& Zettl, R. (2000). Genomics and Proteomics: Functional and Computational Aspects, edited by S. Suhai, pp. 179-189. New York: Kluwer Academic/ Plenum Press.

Helliwell, J. R. (1997). Nature Struct. Biol. 45, 874 876.

Keen, D. A. \& Wilson, C. C. (1996). RAL Technical Report RAL-TR-96-083. http:// www.isis.rl.ac.uk/crystallography/sxd.htm.

Kjems, J., Taylor, A. D., Finney, J. L., Lengeler, H. \& Steigenberger, U. (1997). ESS - A Next Generation Neutron Source for Europe, Vol. IIII. Jülich: ESS Council.

Kossiakoff, A. A. \& Spencer, S. A. (1981). Biochemistry, 20, 6462-6474.

Kuhn, P., Knapp, M., Soltis, S. M., Ganshaw, G., Thoene, M. \& Bott, R. (1998). Biochemistry, 37, 13446-13452.

Langan, P. (1997). Physica B, 234-236, 213-214.

Langan, P., Lehmann, M. S., Wilkinson, C., Jogl, G. \& Kratky, C. (1999). Acta Cryst. D55, 51-59.

Lehmann, M. S., Mason, S. A. \& McIntyre, G. J. (1985). Biochemistry, 24, 5862-5869.

Lehmann, M. S. \& Stansfield, R. F. D. (1989). Biochemistry, 28, 7028-7033.

Mitsch, C. (1998). PhD thesis. Department of Physics, University of Cambridge, England.

Moncton, D. (2000). SNS - Advancing the Frontiers of Science and Technology. Oak Ridge: SNS.

Mueller, U., Muller, Y. A., Herbst-Irmer, R., Sprinzl, M. \& Heinemann, U. (1999). Acta Cryst. D55, 1405-1413.

Mueller, U., Perl, D., Schmid, F. X. \& Heinemann, U. (2000). J. Mol. Biol. 297, 975-988.

Mueller, U., Schübel, H., Sprinzl, M. \& Heinemann, U. (1999). RNA, 5, 670-677.

Myles, D. A. A., Bon, C., Langan, P., Cipriani, F., Castagna, J. C., Lehmann, M. S. \& Wilkinson, C. (1998). Physica B, 241-243, 1122-1130.

Niimura, N. (1996). Neutrons in Biology, edited by B. P. Schoenborn \& R. B. Knott, pp. 415-422. New York: Plenum Press.

Niimura, N., Minezaki, Y., Nonaka, T., Castagna, J. C., Cipriani, F., Hoghoj, P., Lehmann, M. S. \& Wilkinson, C. (1997). Nature Struct. Biol. 4, 909-914.

Nishiyama, Y., Okano, T., Langan, P. \& Chanzy, H. (1999). Int. J. Biol. Macromol. 26, 279-283.

Pace, C. N., Heinemann, U., Hahn, U. \& Saenger, W. (1991). Angew. Chem. Int. Ed. Engl. 30, 343360.

Pebay-Peyroula, E., Garavito, R. M., Rosenbusch, 
J. P., Zulauf, M. \& Timmins, P. A. (1995). Structure, 3, 1051-1059.

Penel, S., Pebay-Peyroula, E., Rosenbusch, J. P., Rummel, G., Schirmer, T. \& Timmins, P. A. (1998). Biochimie, 80, 543-551.

Pope, L. H., Forsyth, V. T., Langan, P., Grimm, H., Rupprecht, A., Denny, R. C. \& Fuller, W. (1998). Physica B, 241-243, 1166-1168.

Pope, L. H., Shotton, M. W., Forsyth, V. T., Langan, P., Denny, R. C., Giesen, U., Dauvergne, M. T. \& Fuller, W. (1998). Physica B, 241-243, 1156-1158.

Reat, V., Patzelt, H., Ferrand, M., Pfister, C., Oesterhelt, D. \& Zaccai, G. (1998). Proc. Natl Acad. Sci. USA, 95, 4970-4975.

Saenger, W. \& Steiner, T. (1998). Acta Cryst. A54, $798-805$.

Sali, A. (1998). Nature Struct. Biol. 5, 1029-
1032.

Sass, H. J., Büldt, G., Gessenich, R., Hehn, D., Neff, D., Schlesinger, R., Berendzen, J. \& Ormos, P. (2000). Nature (London), 406, 649653.

Schoenborn, B. P. (1992). SPIE J. 1737, 234-242. Schoenborn, B. P. (1996). A Protein Crystallography Station at the Los Alamos Neutron Scattering Center, LA-UR 96-3508. Los Alamos: Los Alamos Neutron Scattering Center.

Schoenborn, B. P., Court, J. D., Larson, A. C. \& Ferguson, P. (1999). J. Neutron Res. 7, 89106.

Schoenborn, B. P. \& Knott, R. B. (1996). Neutrons in Biology. New York: Plenum Press.

Shotton, M. W., Pope, L. H., Forsyth, V. T., Langan, P., Denny, R. C., Giesen, U.,
Dauvergne, M. T. \& Fuller, W. (1997). Biophys. Chem. 69, 85-96.

Shu, F., Ramakrishnan, V. \& Schoenborn, B. P. (1996). Neutrons in Biology, edited by B. P. Schoenborn \& R. B. Knott, pp. 309-323. New York: Plenum Press.

Steiner, T. \& Saenger, W. (1993). J. Am. Chem. Soc. 115, 4540-4547.

Waller, D. A. (1989). PhD thesis. Department of Chemistry, University of York, England.

Weik, M., Patzelt, H., Zaccai, G. \& Oesterhelt, D. (1998). Mol. Cell. 1, 411-419.

Weik, M., Zaccai, G., Dencher, N. A., Oesterhelt, D. \& Hauss, T. (1998). J. Mol. Biol. 275, 625634.

Zapp Machalek, A. (2000). NIGMS - Structural Genomics Lay Summary, http://www.nigms.nih. gov/funding/psi/lay_summary.html. 